\title{
Natural hyperbolic dispersion with anisotropic epsilon-near-zero and epsilon-near-pole in squaraine molecular film
}

Minjae Kim Kyu Ri Choi Yeon Ui Lee Benoît Heinrich Soo Young Ko Fabrice Mathevet Jean-Charles Ribierre Anthony D'Aléo* Jeong Weon Wu* Virginie Placide*

Minjae Kim, Kyu Ri Choi

Department of Physics, Ewha Womans University, Seoul 03760, Republic of Korea

Email Address: kimminjea100@naver.com, parily6@naver.com

Prof. Yeon Ui Lee

Department of Physics, Chungbuk National University, Cheongju, Chungbuk 28644, Republic of Korea

Email Address: yeonuilee@cbnu.ac.kr

Prof. Soo Young Ko

Department of Chemistry, Ewha Womans University, Seoul 03760, Republic of Korea

Email Address: sooyko@ewha.ac.kr

Dr. Benoît Heinrich

CNRS-IPCMS, Université de Strasbourg, 23 Rue du Loess, Strasbourg, France

Email Address: benoit.heinrich@ipcms.unistra.fr

Dr. Fabrice Mathevet

Sorbonne Université, Faculté des Sciences, CNRS, Institut Parisien de Chimie Moléculaire (IPCM), UMR 8232, Chimie des Polyméres, 4 Place Jussieu, 75005 Paris, France

Kyushu University, Center for Organic Photonics and Electronics Research, Fukuoka, JP 819-0395, Japan Email Address: fabrice.mathevet@sorbonne-universite.fr

Dr. Jean-Charles Ribierre

Kyushu University, Center for Organic Photonics and Electronics Research, Fukuoka, JP 819-0395, Japan Email Address:

Dr. Anthony D'Aléo

Université de Strasbourg, CNRS, Institut de Physique et Chimie des Matériaux de Strasbourg, UMR

7504, F-67000 Strasbourg, France

Email Address: Anthony.Daleo@ipcms.unistra.fr

Prof. Jeong Weon $\mathrm{Wu}$

Department of Physics, Ewha Womans University, Seoul 03760, Republic of Korea

Email Address: jwwu@ewha.ac.kr

Dr. Virginie Placide

Department of Physics, Ewha Womans University, Seoul 03760, Republic of Korea

Email Address: placidevirginie@gmail.com

Keywords: Hyperbolic dispersion, Epsilon-near-zero, Epsilon-near-pole, Polymethine dye 
Epsilon-near-zero (ENZ) optical material has been employed in a number of novel linear and nonlinear optical applications, owing to the vanishing polarization upon an incident optical wave. In a uniaxial medium possessing hyperbolic energy-momentum dispersion of optical wave, ENZ can take place at ordinary and extraordinary permittivities. Organic thin films presenting a lamellar structure have been reported to exhibit a transverse negative hyperbolic dispersion with ENZ at ordinary permittivity. Here, organic thin film with ENZ at extraordinary permittivity is demonstrated. Newly synthesized polymethine dye (i.e., squaraine indolenine triethyleneglycol molecule) self-organizes to form a layered structure in a pristine film, and both transverse negative and positive hyperbolic dispersions are observed at visible wavelengths. Analysis of tens nanometer-thick pristine film showed that both ENZ and epsilon-near pole (ENP) occur at longitudinal as well as transverse component of dielectric permittivity. Optical characterization of squaraine pristine film is presented, and the importance of transverse positive hyperbolic dispersion in such monolithic thin film is discussed. 


\section{Introduction}

Epsilon-near-zero (ENZ) takes place when the real part of dielectric permittivity crosses the zero-point in the spectrum of dielectric permittivity.[1,2,3] Owing to the dielectric permittivity being near zero, there are a variety of important linear optical applications such as directive emissions, [4] electric field enhancement in ENZ mode,[5] perfect absorption,[6] and a deep direct writing lithography,[7] to name a few. Furthermore, the nonlinear optical processes, both quadratic and cubic, have been found to be enhanced in ENZ spectral range.[8, 9, 10, 11, 12, 13, 14]

When isotropic bulk metal is modelled as free electron gas, Drude permittivity exhibits isotropic ENZ corresponding to the frequency of longitudinal plasma oscillation.[15] Note that ENZ frequency is the same as plasma frequency $\omega_{p}$ in the absence of collision, while introduction of collision causes ENZ to appear below $\omega_{p} .[16]$ Moreover, ENZ frequency of silver $(3.7 \mathrm{eV}, 327 \mathrm{~nm})$ is significantly red-shifted from $\omega_{p}(9.1 \mathrm{eV}, 136 \mathrm{~nm})$, which is attributed to bound electrons in atomic layers adjacent to the metal surface.[17] In deeply subwavelength-thick metal slab, the signature of ENZ permittivity is manifested as plasma resonance absorption at ENZ wavelength for non-normal incident $p$-polarized light, called Ferrell-Berreman mode. $[18,19,20]$

ENZ occurring in inorganic films of transparent conducting oxide[21] such as indium tin oxide[12] and Al-doped $\mathrm{ZnO}[13]$ and organic film of HTS-Squaraine[14] is also isotropic, where enhanced cubic nonlinearity is observed at ENZ wavelength.

ENZ property can be optically anisotropic, and distinct from isotropic ENZ, it has been noted that the presence of symmetry in anisotropic ENZ can be utilized in various optical architectures such as a loss enhanced transmission and collimation, [22] the almost perfect bending waveguides,[23], arbitrary control of energy flux, [24] and the support of quasi-confined ENZ mode.[25] Therefore, it is necessary to explore the structural and optical properties of novel anisotropic ENZ materials.

Hyperbolic metamaterial (HMM) is a highly anisotropic uniaxial ENZ medium possessing hyperbolic energy-momentum dispersion with the relation $\epsilon_{\perp} \cdot \epsilon_{\|}<0$, where $\perp$ (perpendicular and longitudinal) and $\|$ (parallel and transverse) are defined with respect to the film surface with $\hat{z}$ being surface normal. In HMM there always exists a spectral region of dielectric permittivity crossing the zero-point.[26, 27, 28]. Examples of HMM are thin film multilayer composed of alternating layers of metal and dielectric and metallic nanowires in a dielectric host. [29, 30] Natural hyperbolic dispersion is reported in tetradymites of layered structure made of atomic monolayer, [31] and hyperbolic dispersion with anisotropic epsilonnear-pole takes place naturally in Bi-chalcogenides due to the layered structure and extreme structural anisotropy.[32]

Regarding organic thin film, an organic monolithic QQT $(\mathrm{CN})_{4}$ molecular film showed a transverse negative hyperbolic dispersion possessing a transverse ENZ.[33] Recently, regioregular poly (3-alkylthiophenes) (rr-P3AT) organic film is reported to show a similar transverse negative hyperbolic dispersion possessing a transverse epsilon-near-pole (ENP).[34] That is, QQT $(\mathrm{CN})_{4}$ and rr-P3AT films possess ENZ and ENP, respectively, at the parallel component of dielectric constant, i.e., $\epsilon_{\|}$. Hyperbolic dispersion of the above two organic thin films is attributed to a spontaneously aligned lamellar alternating-sublayers structure, similar to polaritons in layered two-dimensional materials and hyperbolic dispersion arising from anisotropic excitons in two-dimensional perovskites. [35, 36]

In this work, we report the demonstration of organic thin film possessing both transverse and longitudinal ENZs. A new squaraine dye, squaraine indolenine triethyleneglycol, is designed to induce oriented anisotropic organization of molecules in a pristine thin film. Squaraine molecules belong to the polymethine family, known to form organic ENZ film.[14] Thin films of newly synthesized dye are prepared in a few tens nanometer thickness and the characteristics of optical properties are assessed.

We have identified that anisotropic thin film of oriented squaraine dye exhibits longitudinal ENZ property, which is evidenced by experiments such as grazing incidence wide angle X-ray scattering (GIWAXS), spectroscopic ellipsometry (SE), attenuated total reflection (ATR) as well as linear optical properties of reflection, transmission, and absorption. Related to ENZ and ENP properties, it is found that squaraine pristine film possesses a hyperbolic dispersion with transverse negative at 570nm - 645nm $\left(\epsilon_{\|}<0\right.$ and $\left.\epsilon_{\perp}>0\right)$ and transverse positive at 645nm -700nm $\left(\epsilon_{\|}>0\right.$ and $\left.\epsilon_{\perp}<0\right)$. 
Those data are compared to finite difference time domain (FDTD) simulation and found to be consistent with the characteristics of longitudinal ENZ property. In particular, associated with transverse positive hyperbolic dispersion, a longitudinal ENZ is observed in squaraine pristine film, which is important in high-resolution imaging application.[37] This is attributed to the characteristic lamellar order of aromatic cores of squaraine dye in successive molecular sublayers in spin-coated molecular film. Also both transverse negative and positive hyperbolic dispersions exhibit ENPs, giving rise to a high refractive in$\operatorname{dex} n \approx 3.7$ near $700 \mathrm{~nm}$.

\section{Design of material and characterization of structure}

\subsection{Design of material}

The molecular structure of squaraine dye possessing short ethylenglycol chain as pendant arm is displayed in Fig. 1. Diethylene glycol chains were chosen in order to observe whether the oxygen atoms could interact in a specific way with fused silica substrate in the squaraine pristine thin film. The alkylation of the 2,3,3-trimethylindolenine was performed in the presence of the tosyl diethylene glycol chain in toluene at $100^{\circ} \mathrm{C}$. This indole intermediate was then condensed onto squaric acid following protocol described in Ref.[38] to give the symmetric squaraine derivative (Fig. 1). The photophysical characterization of the dye in dichloromethane solution was performed and the dye showed the characteristic sharp and intense transition of polymethines dyes at $637 \mathrm{~nm}$ with a full-width-at-half-maximum (FWHM) of $644 \mathrm{~cm}^{-1}$. Fluorescence spectra by exciting at $610 \mathrm{~nm}$, show a typical narrow (FWHM of $690 \mathrm{~cm}^{-1}$ ) emission at $637 \mathrm{~nm}$ with small Stokes Shift $\left(5 \mathrm{~nm}\right.$, i.e., $\left.689 \mathrm{~cm}^{-1}\right)$ characteristic of polymethine dye. See Figure S6 in Supporting Information.

\subsection{Grazing incidence wide angle X-ray scattering}

The organization of squaraine dye in pristine film was investigated by GIWAXS, and 2D pattern is shown in Fig. 2 (a). Molecules were found to self-organize into nanocrystalline domains with spontaneously aligned lamellar structure. This organization obviously results from the nanosegregation of the diethylene glycol substituents and the aromatic cores into alternating sublayers as illustrated in Fig. 2 (b). Successive molecular layers are shifted to each other, leading to an effective lamellar periodicity of two or more layers of thickness $12.2 \AA$. Ratio with calculated molecular volume gives a layer area per molecule of $68 \AA^{2}$ complying with sublayers of compactly arranged, edge-on oriented aromatic cores, and otherwise of interdigitated and loosely packed diethylene glycol chains. The morphology consists in a mosaic of crystallites of 10-20 nm lateral size (as determined from Scherrer equation with shape factor $\mathrm{K}=0.9$ ), oriented with lamellae parallel to film plane and tiled without preferential in-plane orientation.

\section{Characterization of optical properties}

\subsection{Spectroscopic ellipsometry}

Dielectric permittivity spectrum was obtained from SE measurement. According to GIWAXS data and schematic representation in Fig. 2, squaraine backbone in pristine film is standing up with short axis vertical $(\hat{z})$ and long axis horizontal ( $x-y$ plane), resulting in two-dimensional structure corresponding to an optical uniaxial symmetry with extra-ordinary component $\epsilon_{z}=\epsilon_{\perp}$ and ordinary component $\epsilon_{x}=$ $\epsilon_{y}=\epsilon_{\|}$. SE data were fitted by adopting uniaxial anisotropic dielectric permittivity. For details of measurement and modelling, see Experimental Section. Fig. 3 shows dielectric permittivity spectrum with film thickness of $41.5 \mathrm{~nm}$, agreeing with $44 \mathrm{~nm}$ measured by Dektak profiler within $5 \%$.

According to Fig. 3, squaraine pristine film is optically isotropic from UV up to $570 \mathrm{~nm}$. It shows a transverse negative hyperbolic dispersion in the spectral region of $570 \mathrm{~nm}-645 \mathrm{~nm}\left(\epsilon_{\|}<0\right.$ in red solid curve, $\epsilon_{\perp}>0$ in blue solid curve) and a transverse positive hyperbolic dispersion in the spectral region of $645 \mathrm{~nm}$ - 700nm $\left(\epsilon_{\|}>0\right.$ in red solid curve, $\epsilon_{\perp}<0$ in blue solid curve). Extraordinary component $\left(\epsilon_{\perp}\right)$ has ENZ 
at $645 \mathrm{~nm}$ and ENP at $700 \mathrm{~nm}$, while ordinary component $\left(\epsilon_{\|}\right)$has ENZ at 570nm and ENP at $645 \mathrm{~nm}$. Accordingly, ENZ and ENP are anisotropic. Interestingly, ENZ at longitudinal component and ENP at transverse component falls at the identical wavelength of $645 \mathrm{~nm}$.

In contrast to QQT $(\mathrm{CN})_{4}$ and rr-P3AT organic natural hyperbolic films possessing only transverse negative dispersion, $[33,34]$ squaraine pristine film shows both transverse negative and positive hyperbolic dispersions, leading to a presence of ENP at $\epsilon_{\|}$and $\epsilon_{\perp}$, which allows versatile design for photonics device applications. Furthermore, differently from metal-dielectric multilayered structures and metal wire arrays where spectral regions of transverse positive and negative hyperbolic dispersions are widely separated, spectral regions of transverse positive and negative dispersions cross at one wavelength of $645 \mathrm{~nm}$ in squaraine pristine film. From the application point of view, this dual-band hyperbolic dispersion of organic films is essential for broadband wide-angle manipulation of light propagation. This can be attributed to the characteristic lamellar orders of aromatic cores of squaraine molecule in successive molecular sublayers in spin-coated pristine film.

\subsection{Attenuated total internal reflection measurement}

Surface polariton wave is electromagnetic mode propagating at an interface of two optical media with positive and negative permittivities, providing light confinement at subwavelength scale. Similar to surface plasmon polariton (SPP) wave taking place at an interface of air and metallic film, in an organic thin film possessing a spectral region of negative real part of dielectric permittivity a surface exciton polariton (SEP) wave can be excited along an interface of air and organic film.[33, 39, 40] In the latter case, the energy-momentum dispersion relation of $p$-polarized optical wave in glass prism matches with that of SEP, resulting in an ATR resonance.

As discussed above, squaraine pristine films present an oriented layered structure, and at first sight SEP seems to be readily excited at spectral ranges of $\epsilon_{\|}<1$ and $\epsilon_{\perp}<-1$ in Fig. 3. However, at an interface of air and hyperbolic dispersion medium, the relation between air permittivity $\left(\epsilon_{\text {air }}=1\right)$ and $\epsilon_{\perp}$ or $\epsilon_{\|}$of hyperbolic dispersion $\left(\epsilon_{\perp} \cdot \epsilon_{\|}<0\right)$ for surface polariton excitation is quite different from that between air permittivity $\left(\epsilon_{\text {air }}=1\right)$ and isotropic negative permittivity $\epsilon_{i s o}<0$. For an analysis of surface polariton wavevector $\beta$ in terms of $\epsilon_{\perp}$ and $\epsilon_{\|}$, see Supporting Information, where features of ATR resonance at an interface of air and uniaxial hyperbolic dispersion medium are detailed, in particular, Eq. (40) in Supporting Information.

In order to identify properties of hyperbolic dispersion as well as ENZ, SEP wave excitation is examined at the interface of air and squaraine pristine film spin-coated on fused silica substrate. Fig. 4 shows ATR spectra in the spectral range of $570 \mathrm{~nm} \leq \lambda \leq 645 \mathrm{~nm}$ (transverse negative dispersion). In Supporting Information, it is noted that there always exists SEP, regardless of the magnitude of the positive longitudinal permittivity $\epsilon_{\perp}$ as far as $\epsilon_{\|}<0$. We observe that ATR resonances are identified with quality-factor $Q$ depending on the magnitude of negative dielectric permittivity in Fig. 3.

ATR resonance at $650 \mathrm{~nm}$ has a lower $Q$ than ATR resonance at 640nm (Fig. 4 and Fig. 5), which falls on transverse positive and ATR is not excited based on the analysis of surface wave propagating vector $\beta$ (see Supporting Information). It is noted that a real value of $\beta$ exists only when $\epsilon_{\|}>1$.

Fig. 5 shows ATR spectra in the spectral range of $645 \mathrm{~nm} \leq \lambda \leq 700 \mathrm{~nm}$ (transverse positive dispersion), where SEP is excited. A clear ATR resonance is observed at 650nm. It is to be noted that beyond $660 \mathrm{~nm}$, the SEP resonance is broadened owing to a large value of imaginary part of $\epsilon_{\perp}$ as the wavelength increases further.

At the interface between air and an optically anisotropic film, the relation $\epsilon<-1$ of Eq. (43) in Supporting Information does not hold for surface polariton excitation. For example, when photon energymomentum dispersion in the anisotropic film is transverse negative hyperbolic $\left(\epsilon_{\|}<0\right.$ and $\left.\epsilon_{\perp}>0\right)$, a real value of surface polariton wavevector $\beta$ exists even for $-1<\epsilon_{\|}<0$.

By use of Fresnel relation for a uniaxial anisotropic symmetry, [28, 41] we calculated ATR resonance of squaraine pristine film at both transverse negative and positive spectral regions, which is shown in Fig. 6. We find that ATR resonance curves show similar features in Fig. 6, Fig. 4, and Fig. 5. 


\subsection{Linear optical properties}

Fig. 7 shows the measured (black curve) and FDTD simulated (red curve) linear optical spectra of reflection, transmission, and absorption of squaraine pristine film, where FDTD simulation is carried out by using the uniaxial dielectric permittivities obtained from SE measurement of Fig. 3. We also plotted the measurement of linear optical spectra (blue curve) of the film prepared with dye diluted in PMMA matrix, in which the orientational distribution of dye is isotropic. We find that the pristine film has absorption spectrum broadened owing to an aggregation of squaraine dye molecules in pristine film. Spectrum of the imaginary part of ordinary refractive index (dashed pink curve) in Fig. 8 is mainly responsible for the linear absorption spectrum for a normal incidence light ( $\hat{z}$-direction propagating), and the absorption spectrum of UV-Vis measurement in Fig. 7 follows the same feature as the imaginary part of ordinary refractive index (dashed pink curve) in Fig. 8 (c). Here, it is to be noticed that the transition dipole moment of the squaraine is mainly oriented along the long axis of the squaraine backbone with a slight component of this transition dipole moment (induced by the presence of the indolenine) along the short axis (see Figure S7 in Supporting Information). It is interesting to notice that the absorption in $k_{\|}$(in $x$-y plane, parallel to the film surface) presents a random organization resulting in a broad spectrum (Fig. 8). In contrast, the absorption in the $k_{\perp}$ (along $\hat{z}$ direction, perpendicular to the film surface) is well structured with a shoulder at high energy corresponding to an H-like aggregate and a stronger transition at low energy corresponding to a J-like aggregate.[42] These spectra show that there is a sub-organization resulting in a J-like aggregation (with slipped stacks) that can be seen following the $z$-axis (perpendicular plane). This observation can be correlated with the GIWAXS to some extent. It is possible to imagine from Fig. 2 (b) that the formation of nanocrystalline domains in the $x-y$ plane results in random projection of the transition dipole moment on a beam size level. However, while this transition dipole moment being slightly tilted compared to the $x$ - $y$ plane, the $z$ component is similar. See Figure S7 in Supporting Information.

\section{Discussion}

Self-organization of polymethine dyes of squaraine molecule into a layered structure in a pristine film leads to a hyperbolic energy-momentum dispersion of light. Differently from other hyperbolic organic monolithic molecular films, there exists spectral ranges of longitudinal ENZ associated with transverse positive hyperbolic dispersion. Both J-like (centered near 700nm) and H-like (centered near 650nm) aggregates are present, both of which can be found commonly in polymethine dyes. Importantly, slight tilting of transition dipole moment toward surface normal of film plane gives rise to the presence of longitudinal ENZ, namely, in $\epsilon_{\perp}$ associated with J-like aggregate. Narrow J-like band absorption centered near $700 \mathrm{~nm}$ leads to a strong dispersion of refractive index, the maximum reaching $n \approx 3.7$.

ENZ and ENP in squaraine pristine film are in a strong contrast to those in hyperbolic metamaterials of multilayered structure and wire media. Differently from HMM where ENZ and ENP appear in separate components of dielectric permittivity,[43, 44, 45] both ENZ and ENP takes place at longitudinal as well as transverse component of dielectric permittivity.

Natural hyperbolic dispersion with anisotropic ENZ and ENP in squaraine pristine film is similar to that in Bi-chalcogenides, where Gaussian-like dielectric response of inter-band transition is responsible for ENP and anisotropic ENP is attributed to direction-dependent energy-momentum dispersion relation in inherent structural anisotropy.[32]

The presence of longitudinal ENZ and a high refractive index at near IR spectral region makes squaraine pristine film an important optical material for applications such as high-resolution imaging and optoelectronics device employing high refractive index films. The optical dispersion of the synthesized squaraine film exhibits a dual-band hyperbolic dispersion in which both one-sheeted hyperboloid and two-sheeted hyperboloid can exist as the equifrequency surfaces. Therefore, the organic ENZ films can be utilized to manipulate electromagnetic wave propagation in the broadband spectral range. 


\section{Conclusion}

In this work, we introduced a new squaraine dye (i.e., squaraine indolenine triethyleneglycol molecule) exhibiting a promising ENZ property in thin film configuration. Self-organizing in a lamellar structure, the squaraine molecules orient spontaneously perpendicular to the substrate during the film formation leading to pristine films with a strong anisotropy and an uniaxial optical symmetry. Remarkably, it is the report of a longitudinal ENZ using organic dye, which is evidenced by GIWAXS, SE, and ATR measurements.

\section{Experimental Section}

1. Materials and General Techniques

All reagents were purchased from Sigma Aldrich, Acros organics or TCI and were used as supplied without prior purification. Solvent were purchased from Sigma Aldrich or Acros organics as dry or extra dry and stocked under nitrogen atmosphere. For air and moisture sensitive reactions, glasses were dry in oven, and cool down under argon atmosphere. Purifications were done on silica gel flash chromatography (silica gel 60, 230-400 mesh) with the corresponding eluent. TLC were performed on aluminium plates (silica gel $\left.60 \mathrm{~F}_{254}\right)$ from Merck, using an UV lamp $(\lambda=254 \mathrm{~nm}, 315 \mathrm{~nm})$ to visualize the compounds. ${ }^{1} \mathrm{H}$ and ${ }^{13} \mathrm{C}-\mathrm{NMR}$ spectra were recorded on Bruker Ultrashield 300 at respectively $300 \mathrm{MHz}$ and $75 \mathrm{MHz}$. Proton and carbon chemical shifts are reported in ppm $(\delta)$ relative to the signal of the residual protic solvent of $\mathrm{CDCl} 3(\delta 7.27$ and $77.00 \mathrm{ppm}$ for $1 \mathrm{H}$ - and 13C, respectively. Coupling constants $(\mathrm{J})$ are reported in Hertz $(\mathrm{Hz})$, and the following abbreviations are used for signal multiplicities: singlet (s), doublet (d), triplet ( $\mathrm{t})$ and multiplet $(\mathrm{m})$.

\section{GIWAXS}

The characterization of the structural and morphology properties of the spin-coated squaraine thin films was performed by 2D grazing incidence X-ray diffraction experiments at PLS-II 9A U-SWAXS beamline of Pohang Accelerator Laboratory in Korea. The thin films were studied on silicon substrates and the X-rays coming from the in-vacuum Undulator (IVU) were monochromated using $\mathrm{Si}(111)$ double crystals and focused on a detector consisting of a 2D CCD detector (Rayonix SX165). The distance between sample and detector was around $225 \mathrm{~mm}$ and the beam energy was about $11.06 \mathrm{keV}(1.121 \AA)$.

\section{ATR and Optical Characterization}

ATR measurement and analytical calculation are performed in the same manner as described in Supporting Information of Ref.[14]. Linear optical measurements of transmission and reflection are performed with spectrophotometer (Hitachi U-2900). Absorption spectra is obtained from the relation $A=1-R-$ T. Photoluminescence spectra measurement is performed with fluorometer (Horiba Fluoromax-4).

\section{Variable angle spectroscopic ellipsometry}

Variable angle spectroscopic ellipsometry measurement was performed in the reflection mode at three different incidence angle over the spectral range from 381 to $893 \mathrm{~nm}$ using a J. A. Woollam Co., Inc. alphastep SE. Squaraine films were prepared by spin-coating on top of silicon and fused silica substrate. The CompleteEASE ${ }^{\circledR}$ program, which is analysis software, was used for modelling the VASE data. Three reflection data, measured at $65^{\circ}, 70^{\circ}$, and $75^{\circ}$, are obtained for films on silicon and fused silica substrate. The optical parameters were extracted using an (air-film-substrate) model, with squaraine film being uniaxial with the optic axis oriented perpendicular to the plane of the film. Both the in-plane and outplane optical constants were described using Kramers-Kronig consistent parametric functional models for which several complex Gaussian oscillators and Lorentz were used. Mean-square-error (MSE) is 3.046 for 
fitting of squaraine film. See Supporting Information for detailed analysis of modeling and fitting procedure.

\section{Supporting Information}

Supporting Information is available from the Wiley Online Library or from the author.

\section{Acknowledgements}

This work is supported by funding of the Ministry of Science, ICT \& Future Planning, Korea (2014M3A6B3063 2017R1E1A1A01075394). YUL acknowledges National Research Foundation for funding (2021R1F1A1062916). This work was carried out in the framework of CNRSUMI2002 2B-FUEL "Building Blocks for Future Electronics Laboratory". We further thank Pohang Accelerator Laboratory (PAL) for giving us the opportunity to perform the GIWAXS measurements, MEST and POSTECH for supporting these experiments, Dr Hyungju Ahn for adjustments and help, and other colleagues from the 9A USAXS beamline for assistance. Part of this work was also supported by the CNRS (PICS No 8085), France. We thank reviewers for highly valuable comments.

\section{References}

[1] I. Liberal, N. Engheta, Optics and Photonics News 2016, 27, 726.

[2] X. Niu, X. Hu, S. Chu, Q. Gong, Advanced Optical Materials 2018, 6, 101701292.

[3] I. Liberal, N. Engheta, Nature Photonics 2017, 11, 3149.

[4] S. Enoch, G. Tayeb, P. Sabouroux, N. Guérin, P. Vincent, Physical Review Letters 2002, 89, 21 213902.

[5] S. Campione, I. Brener, F. Marquier, Physical Review B 2015, 91, 12121408.

[6] T. S. Luk, S. Campione, I. Kim, S. Feng, Y. C. Jun, S. Liu, J. B. Wright, I. Brener, P. B. Catrysse, S. Fan, et al., Physical Review B 2014, 90, 8085411.

[7] H. Luo, J. Qin, E. Kinzel, L. Wang, Nanotechnology 2019.

[8] M. Lapine, I. V. Shadrivov, Y. S. Kivshar, Reviews of Modern Physics 2014, 86, 31093.

[9] T. S. Luk, D. De Ceglia, S. Liu, G. A. Keeler, R. P. Prasankumar, M. A. Vincenti, M. Scalora, M. B. Sinclair, S. Campione, Applied Physics Letters 2015, 106, 15151103.

[10] A. Capretti, Y. Wang, N. Engheta, L. Dal Negro, ACS Photonics 2015, 2, 111584.

[11] X. Wen, G. Li, C. Gu, J. Zhao, S. Wang, C. Jiang, S. Palomba, C. Martijn de Sterke, Q. Xiong, ACS Photonics 2018, 5, 62087.

[12] M. Z. Alam, I. De Leon, R. W. Boyd, Science 2016, 352, 6287795.

[13] L. Caspani, R. Kaipurath, M. Clerici, M. Ferrera, T. Roger, J. Kim, N. Kinsey, M. Pietrzyk, A. Di Falco, V. M. Shalaev, et al., Physical Review Letters 2016, 116, 23233901.

[14] Y. U. Lee, E. Garoni, H. Kita, K. Kamada, B. H. Woo, Y. C. Jun, S. M. Chae, H. J. Kim, K. J. Lee, S. Yoon, et al., Advanced Optical Materials 2018, 6, 141701400.

[15] C. Kittel, P. McEuen, P. McEuen, Introduction to solid state physics, Wiley New York, 8th edition, 1996.

[16] A. Zangwill, Modern electrodynamics, Cambridge University Press, 2013.

[17] G. Zhu, M. Mayy, M. Bahoura, B. Ritzo, H. Gavrilenko, V. Gavrilenko, M. Noginov, Optics Express 2008, 16, 2015576. 
[18] R. A. Ferrell, Physical Review 1958, 111, 51214.

[19] R. A. Ferrell, E. A. Stern, American Journal of Physics 1962, 30, 11810.

[20] W. D. Newman, C. L. Cortes, J. Atkinson, S. Pramanik, R. G. DeCorby, Z. Jacob, ACS Photonics 2015, 2, 12 .

[21] A. Nemati, Q. Wang, M. Hong, J. Teng, et al., Opto-Electronic Advances 2018, 1, 5180009.

[22] L. Sun, S. Feng, X. Yang, Applied Physics Letters 2012, 101, 24241101.

[23] J. Luo, P. Xu, H. Chen, B. Hou, L. Gao, Y. Lai, Applied Physics Letters 2012, 100, 22221903.

[24] W. Ji, J. Luo, Y. Lai, Optics Express 2019, 27, 1419463.

[25] S. Vassant, J.-P. Hugonin, J.-J. Greffet, Optics Express 2019, 27, 912317.

[26] M. Noginov, M. Lapine, V. Podolskiy, Y. Kivshar, Optics Express 2013, 21, 1214895.

[27] E. E. Narimanov, A. V. Kildishev, Nature Photonics 2015, 9, 4214.

[28] T. Tumkur, Y. Barnakov, S. Kee, M. Noginov, V. Liberman, Journal of Applied Physics 2015, 117, 10103104.

[29] P. Shekhar, J. Atkinson, Z. Jacob, Nano Convergence 2014, 1, 114.

[30] R. Starko-Bowes, J. Atkinson, W. Newman, H. Hu, T. Kallos, G. Palikaras, R. Fedosejevs, S. Pramanik, Z. Jacob, Journal of the Optical Society of America B 2015, 32, 102074.

[31] M. Esslinger, R. Vogelgesang, N. Talebi, W. Khunsin, P. Gehring, S. De Zuani, B. Gompf, K. Kern, ACS Photonics 2014, 1, 121285.

[32] K. C. Maurya, A. Bhui, K. Biswas, B. Saha, Applied Physics Letters 2021, 119, 1011902.

[33] Y. U. Lee, O. P. M. Gaudin, K. Lee, E. Choi, V. Placide, K. Takaishi, T. Muto, P. André, A. Muranaka, M. Uchiyama, F. Mathevet, T. Aoyama, J. Wu, A. D'Aléo, J.-C. Ribierre, ACS Photonics 2019, 6, 71681 .

[34] Y. U. Lee, K. Yim, S. E. Bopp, J. Zhao, Z. Liu, Advanced Materials 2020, 2002387.

[35] T. Low, A. Chaves, J. D. Caldwell, A. Kumar, N. X. Fang, P. Avouris, T. F. Heinz, F. Guinea, L. Martin-Moreno, F. Koppens, Nature Materials 2017, 16, 2182.

[36] P. Guo, W. Huang, C. C. Stoumpos, L. Mao, J. Gong, L. Zeng, B. T. Diroll, Y. Xia, X. Ma, D. J. Gosztola, et al., Physical Review Letters 2018, 121, 12127401.

[37] B. Casse, W. Lu, Y. Huang, E. Gultepe, L. Menon, S. Sridhar, Applied Physics Letters 2010, 96,2 023114.

[38] S. F. Völker, M. Renz, M. Kaupp, C. Lambert, Chemistry-A European Journal 2011, $17,5014147$.

[39] P. Törmä, W. L. Barnes, Reports on Progress in Physics 2014, 78, 1013901.

[40] M. J. Gentile, S. Núñez-Sánchez, W. L. Barnes, Nano Letters 2014, 14, 52339.

[41] M. Noginov, Y. A. Barnakov, G. Zhu, T. Tumkur, H. Li, E. Narimanov, Applied Physics Letters 2009, 94, 15151105.

[42] J. Gierschner, S. Y. Park, Journal of Materials Chemistry C 2013, 1, 375818.

[43] S. A. Ramakrishna, J. Pendry, M. Wiltshire, W. Stewart, Journal of Modern Optics 2003, 50, 9 1419. 
[44] V. Caligiuri, L. Pezzi, A. Veltri, A. De Luca, ACS Nano 2017, 11, 11012.

[45] M. Coppolaro, M. Moccia, V. Caligiuri, G. Castaldi, N. Engheta, V. Galdi, ACS Photonics 2020, 7, 92578. 


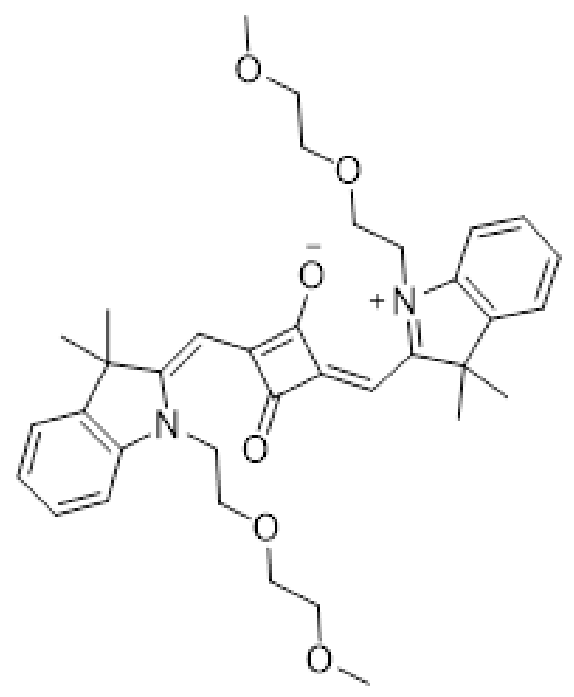

Figure 1: Molecular structure of squaraine molecule synthesized
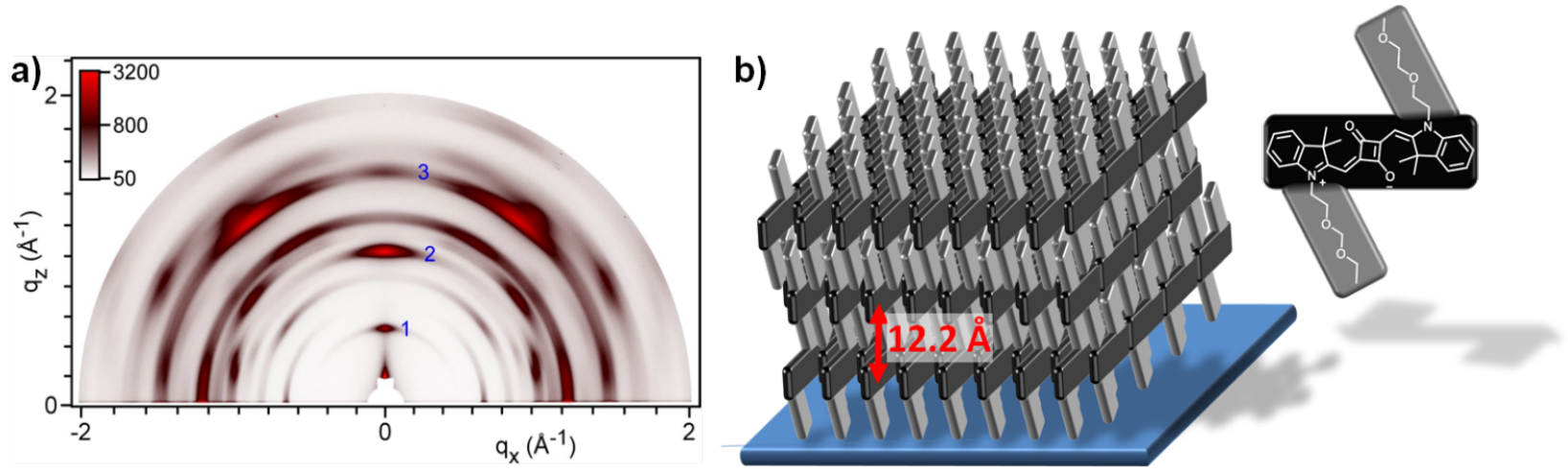

Figure 2: a) Grazing Incidence Wide Angle X-Ray Scattering (GIWAXS) pattern of squaraine dye pristine film that displays a morphology of crystalline lamellae oriented parallel to substrate; as indicated by the lamellar orders lying on the film normal (blue labels). Note that the complete indexation of pattern and the details of the in-plane arrangement would need additional measurements. b) Schematic representation of the spontaneous orientation on the substrate of the lamellar arrangement formed by nanosegregated sublayers of diethylene glycol substituents (in gray) and squaraine aromatic cores (in black). 


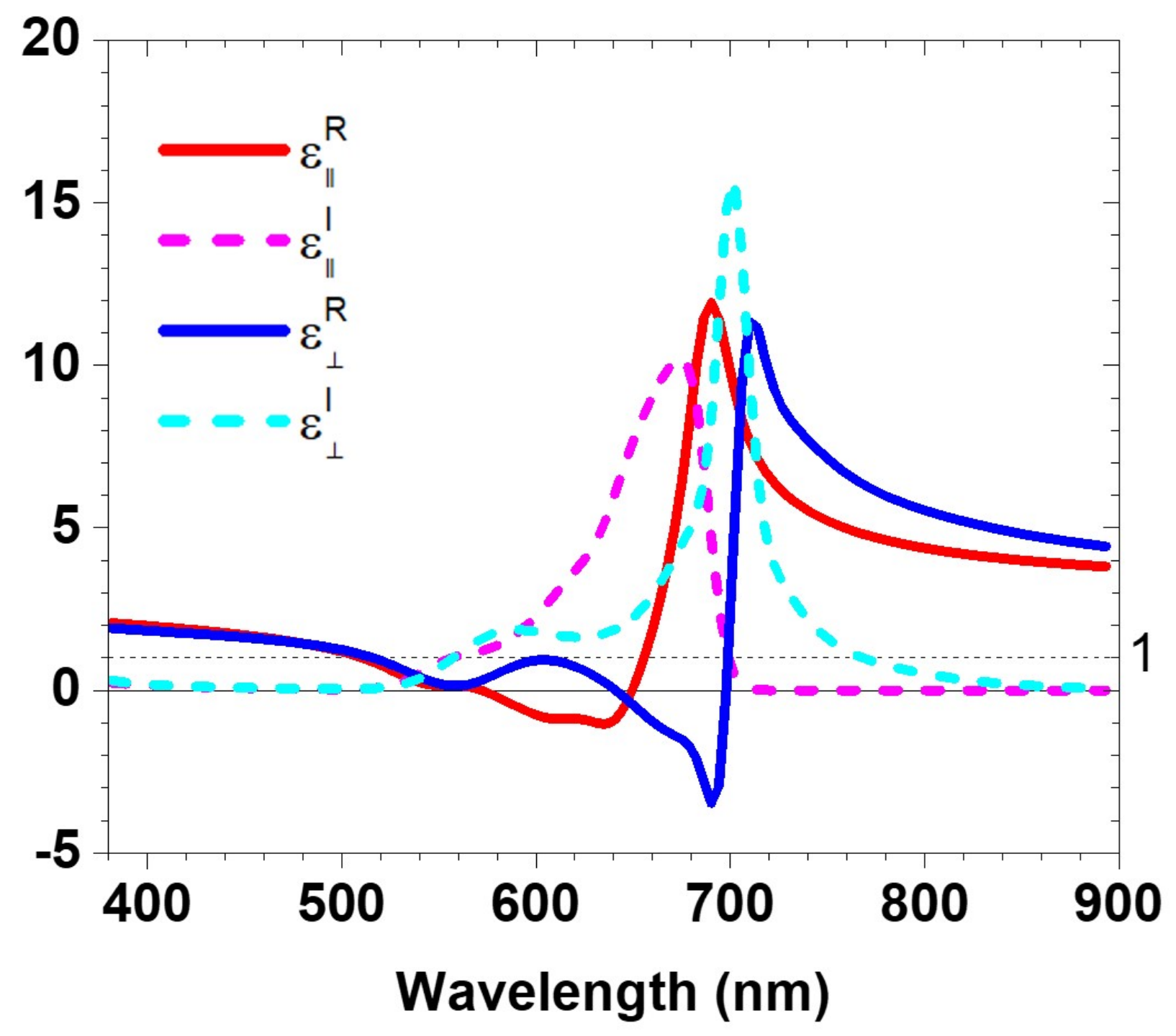

Figure 3: Dispersion of dielectric permittivity obtained from SE measurement is plotted. Blue solid (dashed) curve is real (imaginary) part $\epsilon_{\perp}^{R}\left(\epsilon_{\perp}^{I}\right)$ and ENP is at 700nm where anomalous dispersion crosses zero and ENZ is at $645 \mathrm{~nm}$ where normal dispersion crosses zero. Red solid (dashed) curve is real (imaginary) part $\epsilon_{\|}^{R}\left(\epsilon_{\|}^{I}\right)$ and ENP is at 645nm where anomalous dispersion crosses zero. 

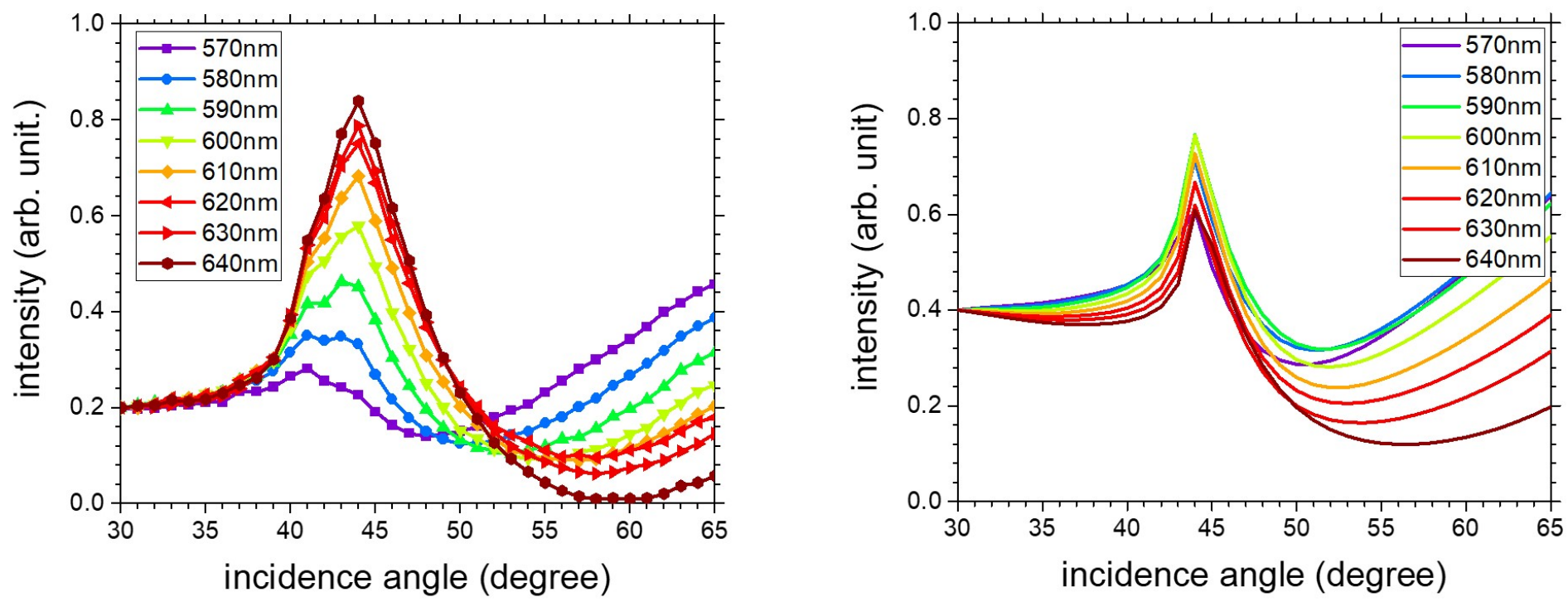

Figure 4: ATR spectra for $570 \mathrm{~nm} \leq \lambda \leq 645 \mathrm{~nm}$ is shown. The left panel is ATR experimental measurement, while the right panel is FDTD simulation.
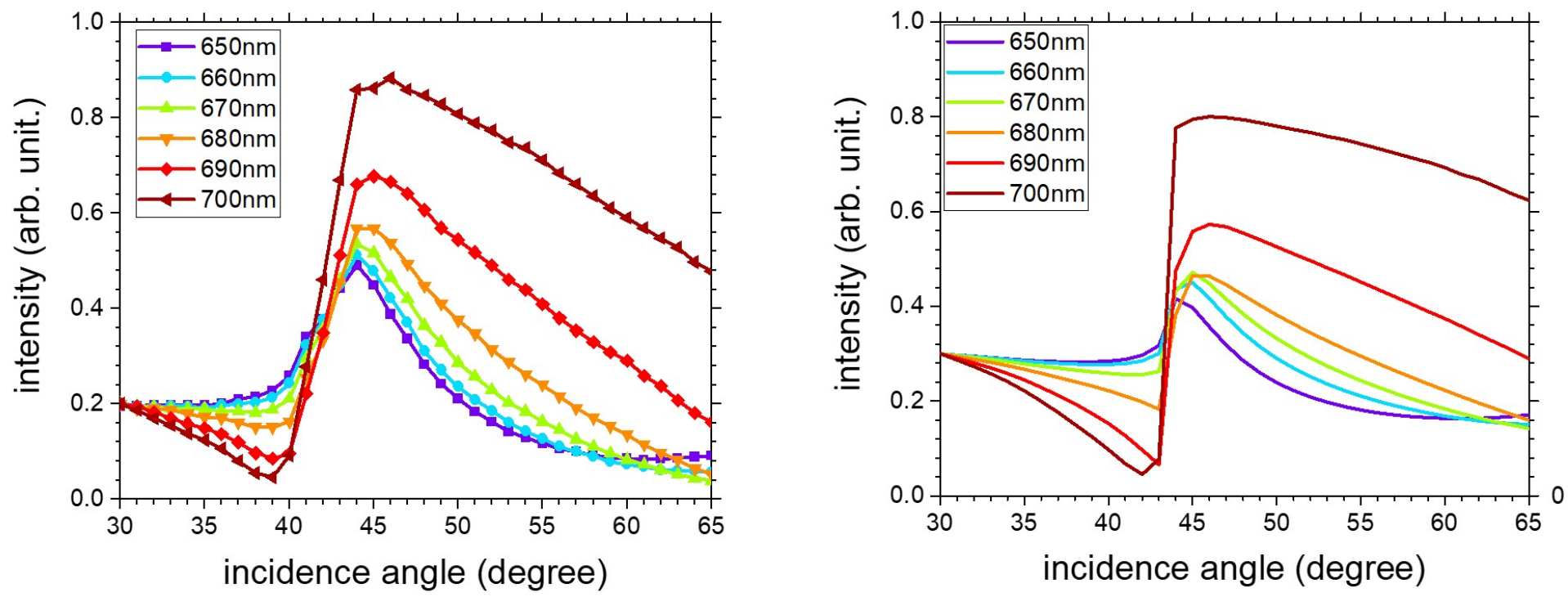

Figure 5: ATR spectra for wavelength $645 \mathrm{~nm} \leq \lambda \leq 700 \mathrm{~nm}$. The left panel is ATR experimental measurement, while the right panel is FDTD simulation. 



Figure 6: Calculations of ATR spectra from $30^{\circ}$ to $60^{\circ}$ are plotted in the spectral range of (a) $570 \mathrm{~nm} \leq \lambda \leq 645 \mathrm{~nm}$ and (b) $645 \mathrm{~nm} \leq \lambda \leq 700 \mathrm{~nm}$.

(a)

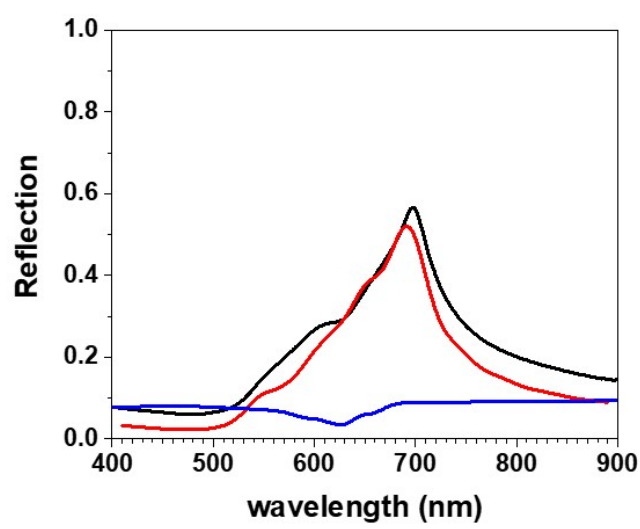

(b)

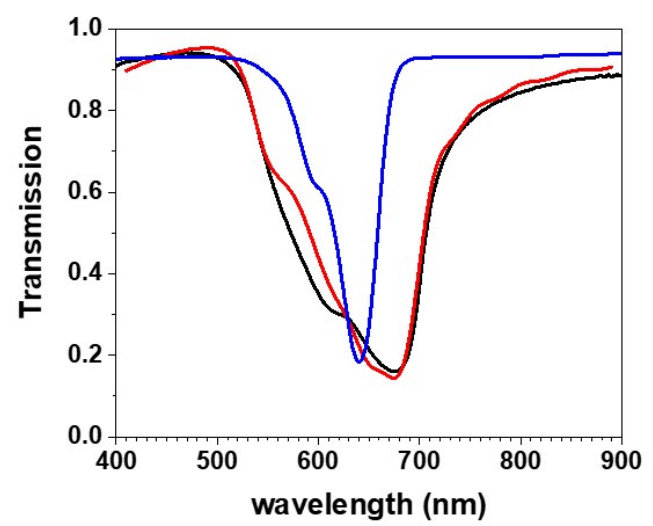

(c)

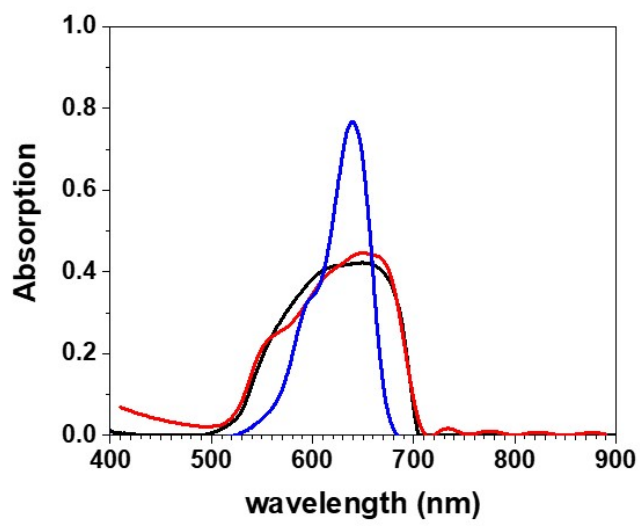

Figure 7: Linear spectra of (a) reflection, (b) transmission, and (c) absorption are plotted. Black, red, and blue curves in each panel correspond to squaraine pristine film, FDTD simulation, and PMMA diluted film, respectively. 


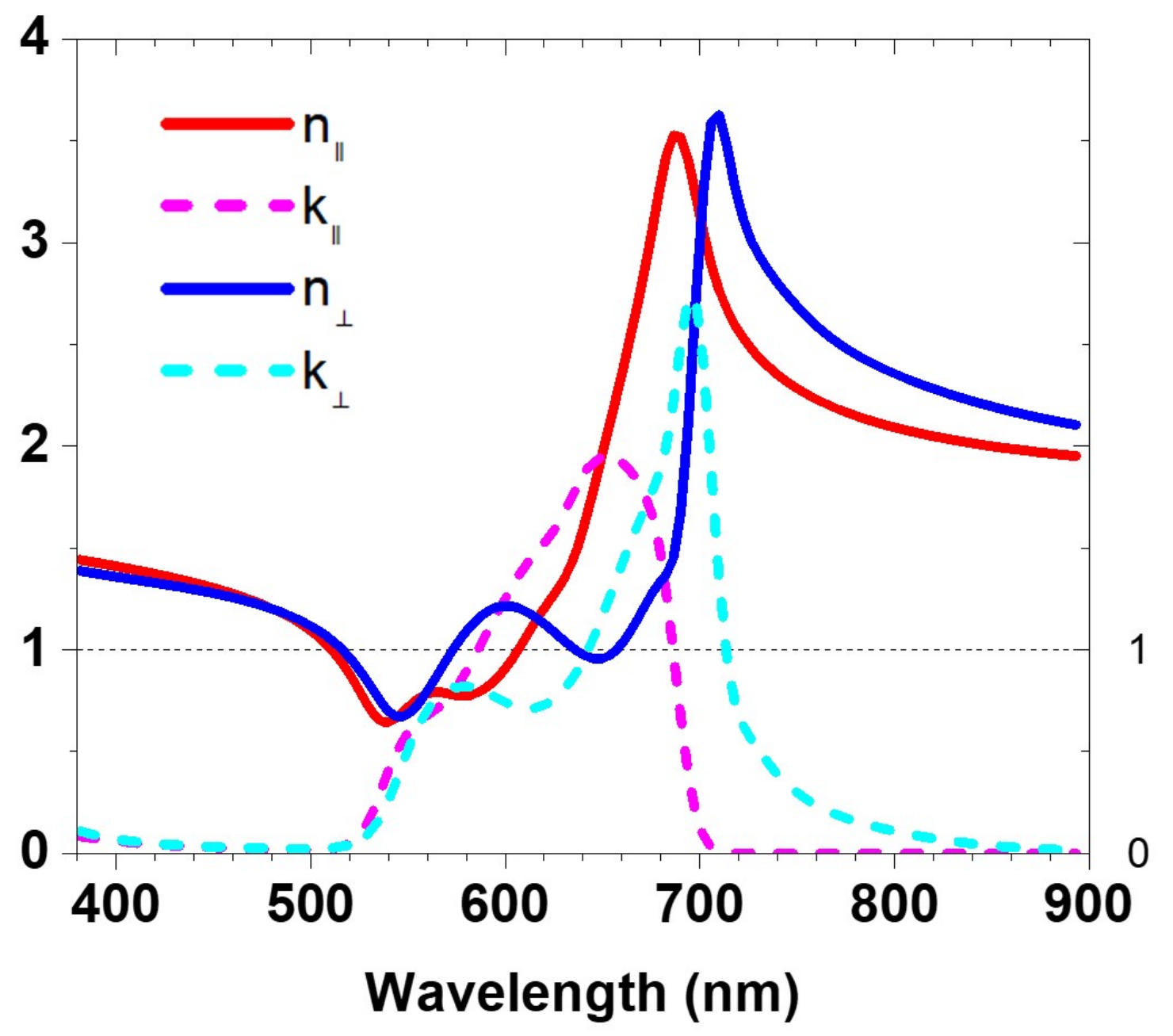

Figure 8: Dispersion of refractive index calculated from dielectric spectrum of Fig. 3 Blue solid (dashed) curve is real (imaginary) part $n_{\perp}\left(k_{\perp}\right)$. Red solid (dashed) curve is real (imaginary) part $n_{\|}\left(k_{\|}\right)$.

Table of Contents

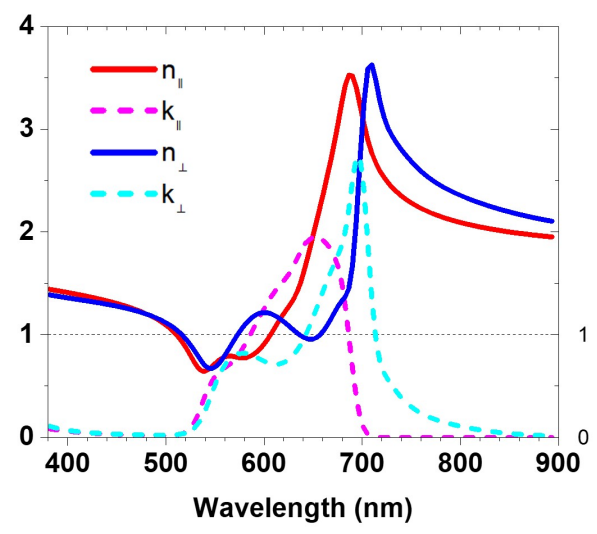

Refractive index 